\title{
Design of a braided composite badminton racket on solidworks
}

Mackenzie Neufeld, Ahmed Samir Ead, Eric Lepp

${ }^{1}$ Department Mechanical Engeneering, University of Alberta

\begin{abstract}
Current badminton rackets are typically made out of steel, aluminum, or carbon fibre. Although these materials perform acceptably, there are some downsides to their properties. However, these non-ideal characteristics of badminton rackets may be overcome with the usage of different manufacturing materials, specifically braided composites. An example of a braided material is Kevlar $\mathbb{R}$. Kevlar is a heat resistant and high strength synthetic fibre that can be manufactured into braids using a maypole braiding system. These Kevlar braids can then be manipulated to a preferred shape for the curing process. In order to come up with a feasible design to base the prototype, a 3D modelling software (SolidWorksTM) is used. This insures geometrical viability and possible to manufacture of the prototype. Modeling a badminton racket on Solidworks required the modelling of a racket head, and handle. The head was created using 2 ellipses, one of which acted as a skeleton, or mold for the Kevlar braid, and the other was a hollow ellipse which encompassed the mold and acted as the Kevlar braid. The solid ellipse was created in two halfs, each having either an extrusion or a hole on the ends. This allowed them to easily attach to form a full ellipse. Once modeled, the solid ellipse was 3D printed to act as the curing mandrel, an internal skeleton for the Kevlar braids. In order to attach the head to the handle, a three part connector piece was created and 3D printed. The rod of the racket was not created with an internal skeleton because the flexibility would falter. Instead, the Kevlar braids were slid off the maderal after curing and attached to the racket heads connector piece. In order to have a balanced weight ratio throughout the racket, the grip was created with an internal 3D printed skeleton. This structure allowed for a feasible, flexible, and strong Kevlar based product.
\end{abstract}

Key words:

Braided Composite, Kevlar, Badminton Racket

Cite as: Neufeld, M., Ead, A.S., Lepp, E. 2019. Design of a Braided Composite Badminton Racket on Solidworks. Alberta Academic Review, Vol 2 (2) 57-58, WISEST Special Issue (non peer-reviewed), DOI 10.29173/aar64. 


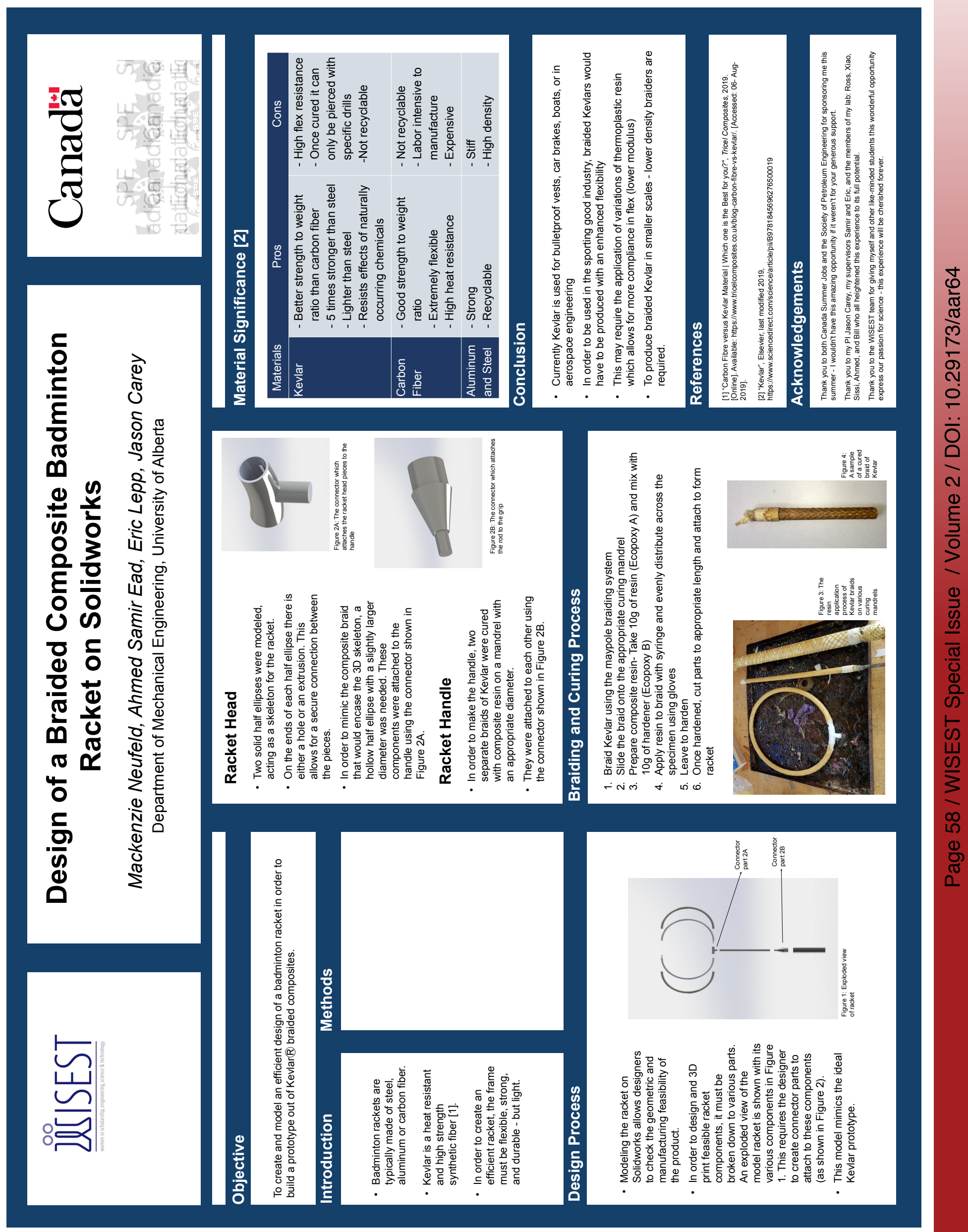

